\title{
Coupling length of silicon-on-insulator directional couplers probed by Fourier-space imaging
}

\author{
J. Jágerská, ${ }^{1, a)}$ N. Le Thomas, ${ }^{1}$ R. Houdré, ${ }^{1}$ D. M. Beggs, ${ }^{2}$ D. O’Brien, ${ }^{2}$ and T. F. Krauss ${ }^{2}$ \\ ${ }^{1}$ Institut de Photonique et d'Electronique Quantiques, Ecole Polytechnique Fédérale de Lausanne \\ (EPFL), Station 3, CH-1015 Lausanne, Switzerland \\ ${ }^{2}$ School of Physics and Astronomy, University of St. Andrews, St. Andrews, Fife, KY16 9SS, United Kingdom
}

(Received 1 February 2008; accepted 26 March 2008; published online 15 April 2008)

\begin{abstract}
We use a Fourier-space imaging technique relying on outcoupling grating probes to study the coupled mode interaction and dispersion properties of guided modes in silicon-on-insulator codirectional couplers. Our approach allows us to measure the mode splitting inherent to coupled systems, determine the mode symmetry, and locally probe the coupling length with an accuracy of $\pm 50 \mathrm{~nm}$. A systematic study of directional couplers with different waveguide widths, coupling gaps, and e-beam exposure doses is reported in order to verify the results across a wider parameter space. (C) 2008 American Institute of Physics. [DOI: 10.1063/1.2909575]
\end{abstract}

Codirectional waveguide couplers are fundamental building blocks of optical integrated circuits. Their primary function is to split or redirect optical signal and, hence, they can be found in all-optical switches, cross connects, tuneable add-drop de/multiplexers, or in a variety of interferometric devices such as Mach-Zehnder interferometers.

State-of-the-art waveguide couplers based on silicon-oninsulator (SOI) substrate offer additional advantage of compactness with coupling lengths of typically $10 \mu \mathrm{m}$ (or even shorter in photonic crystal environment) and propagation losses as low as $2.5 \mathrm{~dB} / \mathrm{cm}^{1-3}$ Their application potential is further promoted by the well-developed complementary metal-oxide semiconductor compatible fabrication technology that allows for mass production of low-cost and highquality devices. It is therefore of high interest to acquire more comprehensive knowledge about such couplers in direct measurement, including coupling lengths, dispersion properties, and critical technological limits.

The physical properties of coupled waveguides have been intensively studied both theoretically and experimentally since the early days of integrated photonics in 1970s. ${ }^{4}$ Along with numerical simulation tools (finite-difference time domain or guided mode expansion methods), analytical coupled mode theory proved suitable for modeling the energy transfer within the coupled systems. ${ }^{4,5}$ In contrast, the experimental verification has been almost exclusively limited to transmission measurements, ${ }^{6}$ which provide spectral information about the coupler performance but cannot directly probe the coupling length or the coupled mode dispersion. So the most important characteristics of the coupler have to be indirectly inferred from a series of measurements, with the precision being affected by imperfections and structural variations across individual samples.

In this paper, we apply an experimental optical technique based on Fourier-space (FS) imaging ${ }^{7}$ to directly probe the fundamental physical interactions within the coupled waveguide structures. The mode splitting of the original isolated waveguide mode is observed in the $k$ space and the dispersion relation of the split modes is studied in detail for different coupler geometries. From the FS images, valuable information about the coupling length/strength and its spectral

${ }^{a)}$ Electronic mail: jana.jagerska@epfl.ch. dependence can be directly reconstructed with high accuracy and without the need for time-consuming repetitive measurements.

Symmetric directional couplers and isolated reference wire waveguides were designed and fabricated in a silicon layer using a SOI wafer $(220 \pm 5 \mathrm{~nm}$ thick Si layer on top of a $2 \mu \mathrm{m} \mathrm{SiO}{ }_{2}$ buffer). A $350 \mathrm{~nm}$ thick layer of ZEP-520A, Zeon Chemicals, Japan, was used as a resist and etch mask. The patterns were generated by electron beam lithography and, following the development of the resist, the pattern was directly transferred into the silicon layer using low power, low dc bias, reactive ion etching with a $\mathrm{SF}_{6} / \mathrm{CHF}_{3}$ gas mix, optimized to yield wire waveguides with vertical sidewalls. ${ }^{8}$ A total of 21 different coupler and waveguide structures with varying wire width $d(350-460 \mathrm{~nm})$ and coupled waveguide separation distance $g(220-390 \mathrm{~nm})$ were prepared from seven distinct mask patterns under different e-beam-dose conditions. A typical top-view scanning electron microscope (SEM) image together with the cross-sectional layout of a final device is shown in Fig. 1(a).

At both sides of the structures, a linear probe grating (LPG) with a period $\Lambda=700 \mathrm{~nm}$ was designed to diffract a small fraction of the guided light into the far field. This light carries amplitude and phase information about the propagating modes, which can be retrieved by use of conventional optics. The waveguide-grating separation distance is $600 \mathrm{~nm}$, ensuring the electric field decay by two orders of magnitude at the LPG. Hence, such separation is large enough not to noticeably influence the loss figure and dispersion properties
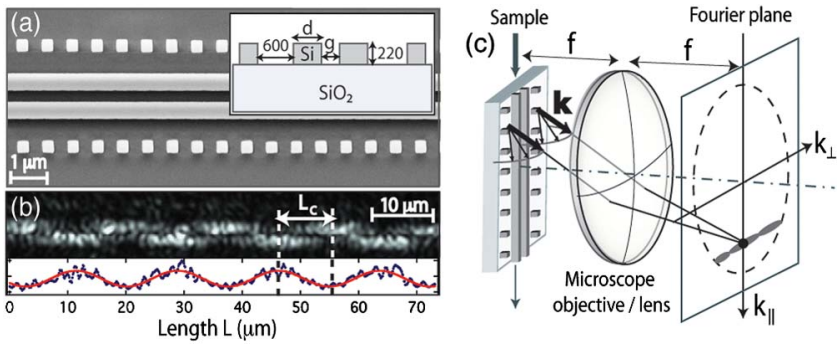

FIG. 1. (Color online) (a) Top-view SEM image and cross-sectional layout (inset) of directional coupler with LPG; dimensions given in nanometer. (b) Corresponding near-field IR image. Below: intensity profile at the bottom LPG. (c) Experimental setup schematically showing the Fourier plane position and the FS image formation. 
of the guided modes, yet at the same time, sufficient to yield a scattered intensity of detectable level.

An external cavity continuous-wave laser diode tuneable within the wavelength range $1480-1650 \mathrm{~nm}$ was used to probe the samples via a lensed fiber and adiabatically tapered access waveguides. $400-\mu \mathrm{m}$-long coupled waveguide section was initiated by symmetric $S$-shaped bends of radius $50 \mu \mathrm{m}$. Polarization of the excitation was set to transversal magnetic (TM), i.e., perpendicular to the sample surface, as stronger mode interaction is observed in this case. The light scattered at the probe gratings was collected from the top of the sample by a high numerical aperture microscope objective $(\mathrm{NA}=0.9)$ and projected onto a near infrared (NIR) camera.

Standard imaging of the sample surface yields a realspace image, which reflects the intensity distribution at the site of the scatterers, i.e., the LPG. As shown in Fig. 1(b), this allows us to map the transfer between the coupled waveguides and estimate the coupling length. In the absence of probe gratings, no intensity pattern can be observed because of the low intrinsic scattering loss of SOI structures and the low spatial resolution of the NIR imaging, which cannot resolve two closely spaced wire waveguides.

By imaging the back focal plane of the collecting lens [Fig. 1(c)], we can access the far-field or $k$-space image, which represents the optical Fourier transform of the nearfield pattern. In such an image, every point $\left[k_{\|}, k_{\perp}\right]$ is uniquely related to a wave vector $k$ of the radiated field. Proper design of the probe gratings ensures that the major contribution to the radiated light comes from the first diffraction order of the guided field scattered at the LPG. According to wave vector conservation, the parallel component of the $k$-vector $k_{\|}$[see Fig. 1(c)] is then strictly related to the propagation constant of the guided modes $\beta$ and takes a single value $k_{\|}=\beta-2 \pi \Lambda$, where $\Lambda$ is the period of the LPG. Hence, by recording $k_{\|}$for each excitation wavelength, we can unambiguously reconstruct the dispersion relation of the guided field. A more detailed discussion about the dispersion measurement technique can be found in Ref. 7.

Figures 2(a) and 2(b) show a far-field image of a wire waveguide and a directional coupler of the same waveguide geometry $(d=380 \mathrm{~nm}, g=250 \mathrm{~nm})$. In both cases, the $k$-space pattern is formed by vertical lines; their position along the $k_{\|}$axis is determined by the propagation constant(s) of the guided field $\left(k_{\|}=\beta-2 \pi / \Lambda\right)$ and the vertical intensity profile is defined by the radiated field distribution perpendicular to the propagation direction.

While the $k$-space image of an isolated waveguide yields a single line shape, the directional coupler figure consists of two closely spaced lines, characterized by different modulation pattern along the vertical $k_{\perp}$ axis. The two lines correspond to two modes of the coupled waveguides, which appear upon splitting of the isolated waveguide mode in the coupled configuration. The magnitude of the mode splitting $\Delta k_{\|}$is a direct measure of the coupling strength, increasing for enhanced interaction between the coupled waveguides. In our experimental configuration, we can determine $\Delta k_{\|}$with a precision of $0.0025 \mu \mathrm{m}^{-1}$. Expressed in terms of the coupling length $L_{C}=\pi / \Delta k_{\|}$, it can be measured with an accuracy of $\pm 50 \mathrm{~nm}$, which is quite remarkable. This accuracy is based on a single wavelength measurement and primarily depends on the signal-to-noise ratio and the field of view of

the imaging lens, and can be further enhanced. depends on the cross-sectional layout of the coupler, in par-
Downloaded 11 Dec 2008 to 128.178.175.90. Redistribution subject to AlP license or copyright; see http://apl.aip.org/apl/copyright.jsp

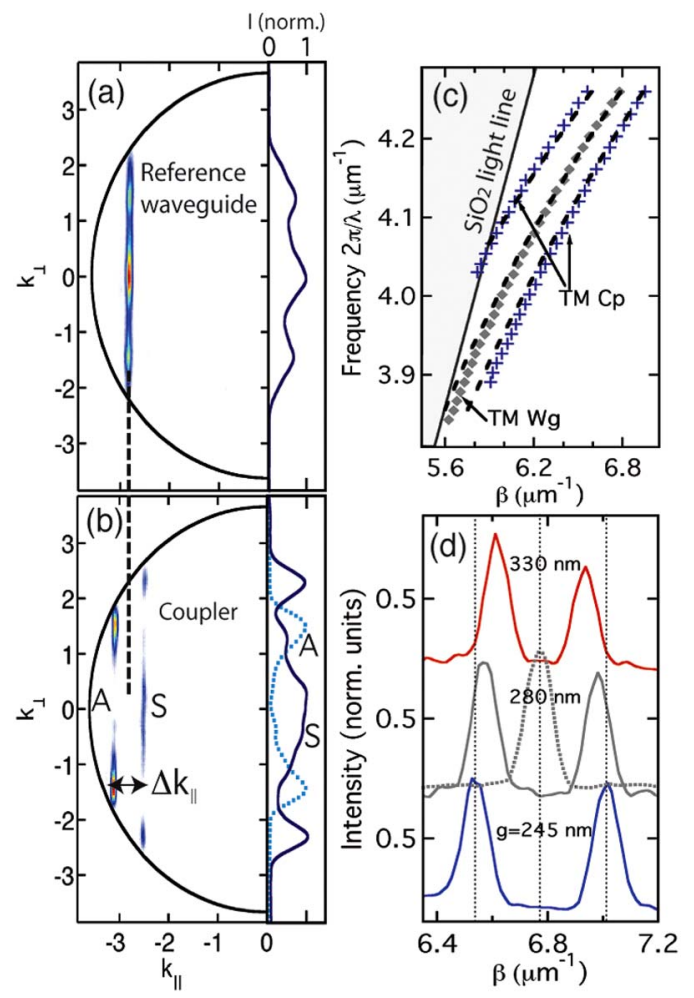

FIG. 2. (Color online) [(a) and (b)] FS image of reference wire waveguide and directional coupler at $\lambda=1550 \mathrm{~nm}$, respectively. Right: corresponding vertical line profiles. (c) Experimental dispersion curves of coupler $(\mathrm{Cp},+)$ and reference wire waveguide $(\mathrm{Wg}, \bullet)$ compared to the theoretical results calculated by CAMFR (dashed line). (d) FS profiles along $k_{\|}$direction shown for constant wire width $d=430 \mathrm{~nm}$ and decreasing waveguide separation $g(\mathrm{Cp}-$ solid, $\mathrm{Wg}-$ dotted line) .

The symmetry character of the measured modes can be equally deduced from the $k$-space observation. As the guided field is diffracted at the two LPGs designed alongside each structure, it produces an interference pattern that is observed in the far field along the $k_{\perp}$ direction. Careful analysis of this pattern allows us to assign the parity of the measured modes. For instance, an intensity maximum of the interference profile at $k_{\perp}=0$ implies that the field at both LPGs is in phase and the mode under investigation is symmetric. Symmetry assignment of the measured modes is indicated in Fig. 2(b), where "A" designates the antisymmetric and " $\mathrm{S}$ " the symmetric modes.

Figure 2(c) shows the measured dispersion diagram of the coupled modes of directional coupler with $d=370 \mathrm{~nm}$ and $g=345 \mathrm{~nm}(+)$ compared to the dispersion curve of the isolated reference waveguide $(\checkmark)$; in both cases, the dispersion curves were obtained by recording the spectral shift of the $k_{\|}$line position in the FS. High precision dispersion measurements result in smooth dispersion curves, which permits to retrieve the spectral dependence of the group index or higher order dispersion. ${ }^{9}$ Dashed lines that are also shown in the figure represent the dispersion curves simulated by the guided-mode-expansion based CAMFR (CAvity Modelling FRamework) solver. ${ }^{10}$ A very good agreement between the theory and experiment is achieved, especially for the frequencies well above the mode cutoff.

The coupling strength and, hence, the magnitude of the mode splitting in codirectional couplers is proportional to the mode field overlap between the individual waveguides. This depends on the cross-sectional layout of the coupler, in par- 

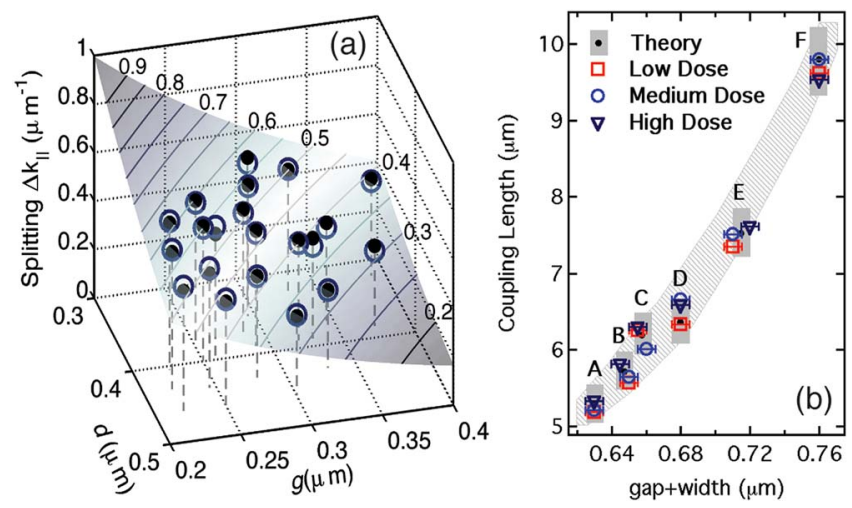

FIG. 3. (Color online) (a) Magnitude of the line splitting $\Delta k_{\|}(d, g)$ at $\lambda=1500 \mathrm{~nm}$. Experimental data $(\bigcirc)$ are compared to the theory $(\bigcirc)$. (b) Experimental and theoretical coupling lengths of different mask designs (labeled as A-F) and e-beam doses (theory: medium e-beam dose only) plotted vs the sum of the wire width $d$ and waveguide separation distance $g$. Theoretical coupling length $L_{c}(d+g)$ is shown as a shaded region.

ticular on the proximity of the two waveguides $g$ and the waveguide width $d$, which is a measure of the lateral mode field confinement. As an example, Fig. 2(d) illustrates an increase in the mode splitting $\Delta k_{\|}$when decreasing the waveguide separation distance from 330 to $245 \mathrm{~nm}$, keeping the constant waveguide thickness of $430 \mathrm{~nm}$.

In order to more closely investigate the relation between the mode splitting $\Delta k_{\|}$and the two structural parameters $g$ and $d$, we use the results of coupled mode theory derived for symmetric slab waveguides ${ }^{11}$ combined with the effective index method. This approach allows us to express the mode splitting function $\Delta k_{\|}(d, g)$ in an analytical form:

$$
\Delta k_{\|}=\frac{\pi}{L_{C}}=\frac{2 \kappa^{2} \gamma \exp (-g \gamma)}{k_{0}^{2} \beta(d)\left(n_{\mathrm{eff}}^{2}-1\right)(d+2 / \gamma)},
$$

where $\kappa=\sqrt{n_{\text {eff }}^{2} k_{0}^{2}-\beta^{2}}, \gamma=\sqrt{\beta^{2}-k_{0}^{2}}, k_{0}$ is the wave vector of the free space, $\beta(d)$ is the $d$-dependent propagation constant of an isolated waveguide, and $n_{\mathrm{eff}}$ is the effective refractive index of an unpatterned SOI membrane.

In Fig. 3(a), the theoretical mode splitting given by Eq. (1) is compared to the experimental data measured for all available coupler designs. In the theoretical model, the effective index of the $220 \mathrm{~nm}$ thick Si device layer $n_{\text {eff }}^{\mathrm{TM}}=2.01$ was used and the propagation constant $\beta(d)=-6.2 d^{2}+9.3 d$ $+3.9\left[\mu \mathrm{m}^{-1}\right], d$ in $\mu \mathrm{m}$ was extrapolated from the experimental data measured on isolated reference waveguides. The two-dimensional function $\Delta k_{\|}(d, g)$ is shown as a surface plot with markers $(\bigcirc)$, which help to visualize the position discrepancy relative to the experimental values $(\mathbf{O})$. An excellent consistency of the measured data and the theoretical results is achieved, demonstrating that the presented analytical model can closely describe the mode splitting in high index contrast SOI coupled systems.

Figure 3(a) further shows that within the studied parameter range, the magnitude of the mode splitting roughly linearly depends on both the wire width $d$ and the waveguide separation distance $g$. The proportionality constants of this dependence are similar for both parameters, which means that if the wire width is increased and the gap thickness reduced by the same amount, the resulting change in the coupling strength is minimal. Hence, the quantity $g+d$ can be considered as a measure of the coupled interaction. This is demonstrated in Fig. 3(b), which shows the analytical plot Downloaded 11 Dec 2008 to 128.178 .175 .90 . Redistribution subject
$L_{C}(g+d)$ for all $g$ and $d$ values found within the studied parameter range (shaded region). The experimental coupling lengths of individual coupler designs are also shown together with the corresponding analytical values calculated for the medium e-beam dose. Rectangles enclosing the theoretical points represent $\pm 5 \mathrm{~nm}$ uncertainty in the coupler lateral parameter specification, reflecting the accuracy with which the dimensions of actual devices can be determined.

The coupling length $L_{c}$ monotonically increases with $g+d$ and for the different designs it ranges from 5 to $10 \mu \mathrm{m}$. Nevertheless, it only weakly depends on the e-beam dose, although the structural parameters considerably vary upon the dose change. Variation in the dose of the e-beam patterning keeps the sum $d+g$ constant and has therefore only a small effect on the resulting coupling length; for all three reported e-beam doses the relative difference in $L_{c}$ lies within the error of the structural parameter specifications, as can be seen in Fig. 3(b). Hence, once the proper mask design is available, the fabrication procedure is robust against small deviations from the target settings.

To conclude, we have noninvasively and accurately determined the coupling length and the dispersion properties of directional couplers based on SOI. Using linear grating probes combined with FS imaging, this was possible in one shot, without the need for repetitive measurement as in the case of commonly used transmission experiments. For TMpolarized excitation, the experimental coupling lengths were found within the range of 5 to $10 \mu \mathrm{m}$, increasing as a onedimensional function of the quantity $d+g$, where $d$ represents the waveguide width and $g$ the gap separating the coupled waveguides. A systematic study of different coupler designs also revealed a low sensitivity of the coupling length to the e-beam dose used upon fabrication. Beyond, it should be noted that due to local nature of LPG probes, the presented technique allows for on-chip measurements of integrated devices or more advanced systems, e.g., dynamic switches or coupled slow light photonic crystal waveguides, where the increasing losses make the transmission measurements difficult.

This work was supported from the European projects ePIXnet (IST-004525), Funfox (IST-004582), the COST P11 action, and the Swiss NCCR-Quantum Photonics. The authors want to thank Vasily Zabelin for valuable scientific discussions.

${ }^{1}$ H. Yamada, T. Chu, S. Ishida, and Y. Arakawa, IEICE Trans. Electron. E90-C, 59 (2007).

${ }^{2}$ D. M. Beggs, T. P. White, L. O'Faolin, and T. F. Krauss, Opt. Lett. 33, 147 (2008).

${ }^{3}$ P. Dumon, G. R. A. Priem, L. R. Nunes, W. Bogaerts, D. Van Thourhout, P. Bienstman, T. K. Liang, M. Tsuchiya, P. Jaenen, S. Beckx, J. Wouters, and R. Baets, Jpn. J. Appl. Phys., Part 1 45, 6589 (2006).

${ }^{4}$ D. Marcuse, Theory of Dielectric Optical Waveguides (Academic, New York, 1974).

${ }^{5}$ A. Yariv, IEEE J. Quantum Electron. QE-9, 919 (1973).

${ }^{6}$ H. Yamada, T. Chu, S. Ishida, and Y. Arakawa, IEEE Photonics Technol. Lett. 17, 585 (2005).

${ }^{7}$ N. Le Thomas, R. Houdré, M. V. Kotlyar, D. O’Brien, and T. F. Krauss, J. Opt. Soc. Am. B 24, 2964 (2007).

${ }^{8}$ L. O'Faolain, X. Yuan, D. McIntyre, S. Thoms, H. Chong, R. M. De la Rue, and T. F. Krauss, Electron. Lett. 42, 1454 (2006).

${ }^{9}$ J. Jágerská, N. Le Thomas, R. Houdré, J. Bolten, C. Moormann, T. Wahlbrink, J. Čtyroký, M. Waldow, and M. Först, Opt. Lett. 32, 2723 (2007).

${ }^{10} \mathrm{P}$. Bienstman, Rigorous and Efficient Modeling of Wavelength Scale Photonic Components, Ph.D. thesis, Ghent University, 2000.

${ }^{11}$ D. Marcuse, J. Lightwave Technol. LT5, 113 (1987). 